\title{
新型变排量外啮合齿轮洜流量特性研究*
}

\author{
赵 斌 何炎泳 郭伟伟 郝云晓 权 龙 \\ (太原理工大学新型传感器与智能控制教育部/山西省重点实验室 太原 030024)
}

\begin{abstract}
摘要: 齿轮洜具有结构紧凑、体积小、转速范围大和耐冲击性能强等优点, 作为动力元件广泛应用于液压系统。然而, 与柱 塞葲和叶片洜相比, 齿轮葲难以实现变量控制, 限制了其应用范围。为此, 提出一种变滑块位置一困油区域的齿轮百变量方 式。对变量齿轮泵进行理论分析, 通过联合仿真和流场可视化仿真详细分析变量齿轮原的特性; 建立试验台对理论和仿真结 果进行了验证。研究结果表明, 通过改变变量模块位置, 调整困油区域, 齿轮原排量可在 $88 \% \sim 100 \%$ 变化; 研究工作将齿 轮百的变量设计提供帮助。
\end{abstract}

关键词: 变量齿轮葲; 流量脉动; 变量滑块

中图分类号: TH137

\section{Study on Flow Characteristics of New Variable Displacement External Meshing Gear Pump}

\section{ZHAO Bin HE Yanyong GUO Weiwei HAO Yunxiao QUAN Long}

(Key Lab of Advanced Transducers and Intelligent Control System of Ministry of Education and Shanxi Province, Taiyuan University of Technology, Taiyuan 030024)

\begin{abstract}
Gear pump is widely used in hydraulic system due to compact structure, small volume, large speed range and strong impact resistance. However, compared with piston pump and vane pump, gear pump is difficult to achieve variable control, which limits its application. Therefore, a variable displacement mode of gear pump with variable slider position-trapped area is proposed. The theory of variable gear pump is analyzed, and the characteristics of variable gear pump are analyzed through joint simulation and flow field visualization simulation. Finally, a test-bench is established to verify the theory and simulation results. The results show that the displacement of gear pump can vary from $88 \%$ to $100 \%$ by changing the position of variable module and adjusting the trapped area. The research work will help the variable design of gear pump.
\end{abstract}

Key words: variable gear pump; flow fluctuation; variable slider

\section{0 前言}

齿轮洜作为液压源, 广泛应用于采矿设备、治 金设备、建筑机械、工程机械、农林机械等领域, 具有结构紧凑、体积小、转速范围大、耐冲击性能 强等优点。在齿轮洜中, 主动轮驱动从动轮, 通过 齿轮啮合, 完成吸油和排油。由于结构限制, 齿轮 泵很难实现变量控制, 限制了齿轮泵的应用范围。

目前, 齿轮百的研究主要集中在数值仿真 ${ }^{[1-2] 、}$ 结构优化和变量控制等方面。在数值仿真方面, FROSINA 等 ${ }^{[3]}$ 建立了齿轮原的三维流体动力学模

* 国家青年科学基金(51605322)、山西省重点研发计划(201803D121098) 和山西省回国留学人员科研(2017-033)资助项目。20190829 收到初稿, 20191005 收到修改稿
型, 用于计算洜容积效率, 并通过试验进行了验证。 CAMPO 等 ${ }^{[4]}$ 等对齿轮洜的气蚀效应进行了数值分 析, 并采用图像测速法分析进出口压力对容积效率 的影响。VACCA 等 ${ }^{[5]}$ 等介绍了用非牛顿流体操作的 外齿轮原的建模技术; 并使用 Hygesim 软件从流量、 压力和效率方面分析齿轮葲的性能 ${ }^{[6]}$ 。CASOLI 等 ${ }^{[7]}$ 使用 Amesim 搭建了外啮合齿轮洜模型, 可用于对 每个齿腔的压力变化进行仿真分析。YOON 等 ${ }^{[8]}$ 采 用浸没固体法，对齿轮洜在不同工况下的运行情况 进行了仿真分析。MUCCHI 等 ${ }^{[9]}$ 建立了一种用于预 测外啮合齿轮洜动态特性非线性集动-弹性动力学 模型。

齿轮葲结构优化主要用于降低噪声、困油冲击 以及流量压力脉动等。在降低噪声方面, MUCCHI 等 ${ }^{[10]}$ 通过融合总参数模型、有限元模型和边界元模 
型对齿轮洜洜的内部、外部噪声进行研究, 为降低 噪声提供了理论依据。阮学云等 ${ }^{[11]}$ 和杜沙沙 ${ }^{[12]}$ 也通 过相应的方法对齿轮洜的噪声机理进行了研究, 并 提出了改进措施。高彦军等 ${ }^{[13]}$ 采用 Fluent 软件对齿 轮泵的流场进行仿真, 分析油液压缩性、黏度等对 流量和压力脉动、噪声的影响。由于齿轮的啮合作 用, 齿轮洜普遍存在困油现象, 困油不仅降低了齿 轮百的容积效率, 而且影响齿轮泵寿命 ${ }^{[14-15]}$ 。李 玉龙等 ${ }^{[16]}$ 和吴小锋等 ${ }^{[17]}$ 对影响齿轮泵困油压力的 因素进行了分析。喊克江等 ${ }^{[18]}$ 通过对齿轮百工作原 理的分析, 提出一种降低齿轮泵困油压力的卸荷 降压槽法, 并对其工作原理及特性进行研究。齿 轮百的流量脉动 ${ }^{[19-20]}$ 较大, 在高精度控制领域应 用受到限制。CASTILLA 等 ${ }^{[21]}$ 建立了完整的外啮 合齿轮洜三维模型, 采用黏性壁法模拟啮合接触 点, 较二维仿真模型具有更准确的流量脉动模拟 结果。李阁强等 ${ }^{[22]}$ 设计了一种双圆弧斜齿轮䈋, 结果表明该齿形可降低齿轮百的流量脉动。孙浩 林 ${ }^{[23]}$ 以圆弧齿轮泵为对象, 基于数学方法建立圆 弧齿形的数学模型, 发现圆弧齿形可以有效减少 流量脉动。

由于齿轮百变量困难, 虽然对变量齿轮泵 ${ }^{[24-25]}$ 的研究由来已久, 但相关文献很少。杨德贵等 ${ }^{[26]}$ 在 专利提出一种径向移动变量齿轮百, 通过使主从动 齿轮轴距变化达到变量效果, 然而, 径向移动的变 量方式制造难度大, 泄漏严重。WINMILL ${ }^{[27]}$ 提出 一种轴向移动变量齿轮百, 通过特制的齿轮套改变 齿轮啮合的厚度而改变齿轮排量, 但这种方式使㬌 整体体积较大, 而且泄漏较大。闻徳生等 ${ }^{[28]}$ 为使定 量齿轮泵输出多级流量, 建立了一种具有多个齿轮 组的齿轮百, 通过改变齿轮洜工作数量实现齿轮洜 多个排量的改变, 无法实现无级变量。

为此, 本文提出一种齿轮百变量方法, 通过调 节齿轮洜困油区, 实现齿轮泵排量小范围调节。研 究中, 开发了基于 SimulationX 软件的变量齿轮泵的 机液联合仿真模型(宏观特性)以及基于 Pumplinx 软 件的流场仿真模型(微观特性), 充分结合 SimulationX 仿真速度快及 pumplinx 微观仿真的优点, 对变量齿 轮洜特性进行了深入分析研究, 并通过试验对所提 变量原理进行了验证。本研究采用的宏观特性和微 观特性仿真结合的方法, 可为液压㫤的仿真研究提 供一条新途径。

\section{1 变量齿轮百原理}

齿轮泵运行过程中, 可分为低压区、过渡区、
高压区三部分, 图 1 为齿轮㫤工作区域的分布情况。 齿轮腔在低压区体积增大, 压力降低, 完成吸油; 齿轮腔在过渡区体积保持不变; 齿轮腔在高压区体 积变小, 压力升高, 向排油区排油。

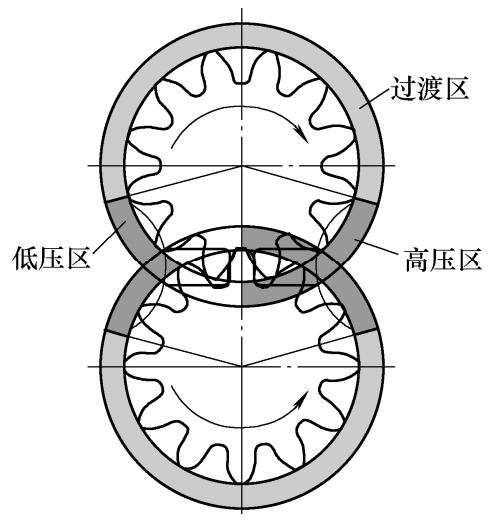

图 1 齿轮洜工作原理

本文提出一种改变困油区体积实现齿轮洜排量 小范围调节的方法, 图 2 所示为变量原理。

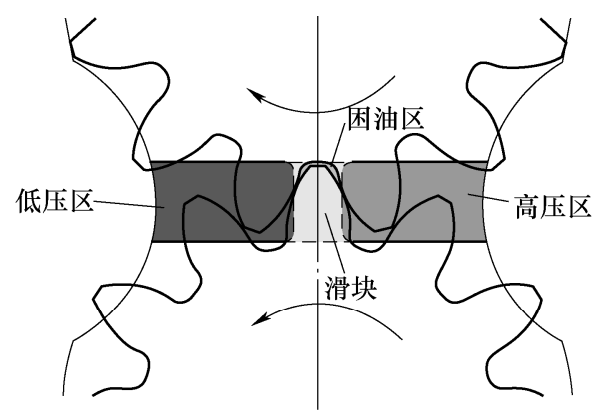

(a) 中位困油区

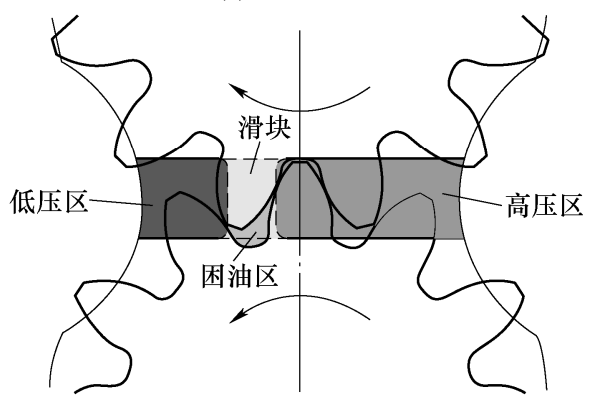

(b) 极限位困油区

图 2 中位与极限位困油区位置、大小及滑块的位置

改变困油区体积可改变齿轮泵排量。滑块处 于图 2a 中位时, 齿轮腔只从低压区吸油, 同样 也仅在高压区排油。当滑块位置处于图 $2 b$ 所示极 限位置时, 齿轮腔容积增大时, 将先从高压区吸 油, 再从低压区吸油, 从而使齿轮㬌排出油液发 生变化。其中，中位的选取原则是困油区体积最 小时的位置, 极限位的选取原则是轮齿不脱离啮 合条件下，困油区体积最大时的位置。改变滑块 相对位置, 可控制齿轮腔从高压区中吸入的油液 体积, 达到变量效果。滑块的设计原则基于齿轮 
轮齿设计, 基本要求是轮齿啮合过程中切分吸排 油区, 使两区域不相连, 同时, 使滑块的宽度最 小化以增大变量效果, 据此, 设计矩形滑块作为 参考使用。

\section{2 理论分析与机液联合仿真}

本节通过齿轮泵理论和仿真分析为变量齿轮葲 提供理论依据, 表 1 所示为采用的齿轮泵中的主动 齿轮与从动齿轮的参数。

\section{表 1 齿轮参数}

\begin{tabular}{lc||lc}
\hline \multicolumn{1}{c|}{ 参数 } & 数值 & \multicolumn{1}{c}{ 参数 } & 数值 \\
\hline 法面模数 $/ \mathrm{mm}$ & 2.75 & 主动轮齿宽 $/ \mathrm{mm}$ & 27 \\
端面模数 $/ \mathrm{mm}$ & 2.75 & 从动轮齿数 & 12 \\
法面压力角 $/\left({ }^{\circ}\right)$ & 17.5 & 从动轮变位系数 & -0.36 \\
螺旋角 $/\left({ }^{\circ}\right)$ & 0 & 从动轮齿宽 $/ \mathrm{mm}$ & 27 \\
主动轮齿数 & 12 & 中心距 $/ \mathrm{mm}$ & 33 \\
主动轮变位系数 & 0.36 & 主动轮分度圆直径 $/ \mathrm{mm}$ & 33 \\
\hline
\end{tabular}

图 3 所示为齿轮百工作过程中各工作区域的划 分示意图, 可为后期的理论和仿真分析提供基础。 表 2 所示为对图 3 中各符号的解释。

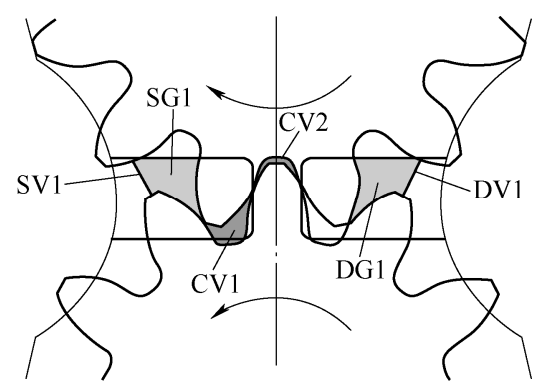

图 3 齿轮百各工作区域划分

表 2 符号意义

\begin{tabular}{cl}
\hline 符号 & \multicolumn{1}{c}{ 参数名称 } \\
\hline CV1(或 CV2) & 齿轮腔容积大小 \\
DV1(或 DV2) & 齿轮腔顶与排油腔相连的面积 \\
SV1(或 SV2) & 齿轮腔顶与吸油腔相连的面积 \\
DG1(或 DG2) & 齿轮腔侧与排油区卸荷槽相连的面积 \\
SG1(或 SG2) & 齿轮腔侧与吸油区卸荷槽相连的面积 \\
\hline
\end{tabular}

\section{1 理论分析}

图 4 所示为齿轮计算参数图, 取齿廓上两点, 这两点的半径分别为 $r_{\mathrm{t}}$ 和 $r_{\mathrm{d}}$, 两段圆弧与齿廓围成 面积为 $S_{\mathrm{G}}$, 两点半径与齿廓围成面积为 $S_{\mathrm{inv}}, r_{\mathrm{d}}$ 圆弧 对应圆心角为 $\varphi\left(r_{\mathrm{d}}\right)$, 其计算公式为

$$
\begin{gathered}
S_{\text {inv }}\left(r_{\mathrm{i}}, r_{\mathrm{d}}\right)=r_{\mathrm{b}}^{2}\left(\tan ^{3} \alpha_{\mathrm{t}}-\tan ^{3} \alpha_{\mathrm{d}}\right) / 6 \\
S_{\mathrm{G}}\left(r_{\mathrm{t}}, r_{\mathrm{d}}\right)=(0.5 s / r+\operatorname{inv} \alpha)\left(r_{\mathrm{t}}^{2}-r_{\mathrm{d}}^{2}\right)- \\
\left(\operatorname{inv} \alpha_{\mathrm{t}} r_{\mathrm{t}}^{2}-\operatorname{inv} \alpha_{\mathrm{d}} r_{\mathrm{d}}^{2}\right)+2 S_{\text {inv }}\left(r_{\mathrm{i}}, r_{\mathrm{d}}\right)
\end{gathered}
$$

$$
\varphi\left(r_{\mathrm{d}}\right)=s / r-2\left(\operatorname{inv} \alpha_{\mathrm{d}}-\operatorname{inv} \alpha\right)
$$

式中, $s, r$ 为分度圆处的齿厚与半径， $r_{\mathrm{d}}$ 为基圆半 径， $\alpha 、 \alpha_{\mathrm{t}} 、 \alpha_{\mathrm{d}}$ 为 $r 、 r_{\mathrm{t}}$ 和 $r_{\mathrm{d}}$ 处压力角, inv $\alpha 、 i n v \alpha_{\mathrm{t}}$ 、 inv $\alpha_{\mathrm{d}}$ 表示各压力角的渐开线函数。

图 5 所示为齿轮泵困油区示意图。设啮合点 1 与节点 8 之间长度用 $f$ 表示, 困油面积 $S_{0}$ 为齿廓上 点 $1 、 6 、 5 、 2 、 3 、 4$ 所围面积。则

$$
\begin{gathered}
S_{0}=S_{17234}-S_{19256}-S_{1927} \\
S_{17234}=\pi\left(r_{1,1, \mathrm{a}}{ }^{2}-r_{\mathrm{b}}{ }^{2}\right) / z-S_{G}\left(r_{1,1, \mathrm{a}}, r_{\mathrm{b}}\right)+ \\
{\left[\pi / z-0.5 \varphi\left(r_{\mathrm{b}}\right)\right]\left(r_{\mathrm{b}}{ }^{2}-{r_{\mathrm{f}}}^{2}\right)} \\
S_{19256}=S_{G}\left(r_{\mathrm{a}}, r_{1,2, \mathrm{a}}\right) \\
S_{1927}=0.5\left[\varphi\left(r_{1,2, a}\right)-\sin \left(r_{1,2, \mathrm{a}}\right)\right] r_{1,2, \mathrm{a}}{ }^{2}+0.5 \\
{\left[2 \pi / z-\varphi\left(r_{1,1, \mathrm{a}}\right)-\sin \left(2 \pi / z-\varphi\left(r_{1,1, \mathrm{a}}\right)\right)\right] r_{1,1, \mathrm{a}}{ }^{2}}
\end{gathered}
$$

式中, $r_{1,1, \mathrm{a}}=\sqrt{0.25 A^{2}+f_{\mathrm{a}}^{2}-A f_{\mathrm{a}} \sin \alpha_{\mathrm{n}}}, r_{1,2, \mathrm{a}}=$ $\sqrt{0.25 A^{2}+f_{\mathrm{a}}^{2}-A f_{\mathrm{a}} \sin \alpha_{\mathrm{n}}}, z$ 为齿数, $r_{\mathrm{a}} 、 r_{\mathrm{f}}$ 为顶圆 半径、根圆半径, $r_{1,1, \mathrm{a}} 、 r_{1,2, \mathrm{a}}$ 为点 1 到圆心 $O_{1} 、 O_{2}$ 距离, $A$ 表示实际中心距, $\alpha_{\mathrm{n}}$ 为啮合角。

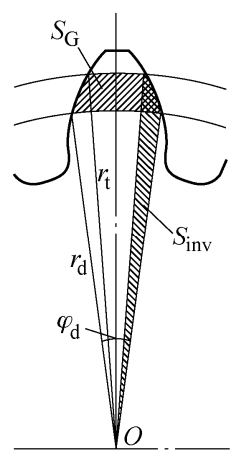

图 4 齿轮计算参数图

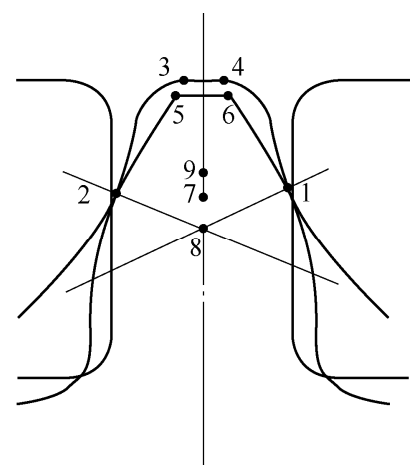

图 5 困油区示意图
由此, 可得困油区的面积表达式

$$
S_{\text {trap }}(f)=S_{0}+\pi\left(f-f_{\mathrm{a}}\right)^{2} / z
$$

齿轮百每一对齿轮腔可视为一单独模块, 因此, 可得齿轮泵排量

$$
V=2 z B\left[S_{\mathrm{M}}-S_{\text {trap }}(f)\right]
$$

式中, $V$ 表示齿轮泵的排量, $B$ 为齿轮厚度, $S_{\mathrm{M}}$ 为 齿轮腔最大面积 $(\mathrm{CV} 1)$ 。

图 6 所示为齿轮洜旋转一周, $\mathrm{CV} 1$ 及 $\mathrm{CV} 2$ 变化 曲线(一个周期)。CV1 的最大面积为 $30.13 \mathrm{~mm}^{2}$, 困 油区位于中位时面积 $1.6 \mathrm{~mm}^{2}$, 而困油区位于极限 位置时面积为 $4.94 \mathrm{~mm}^{2}$ 。

由式(1)计算可得困油区中位时的排量为 $18.49 \mathrm{~mL} / \mathrm{r}$, 而困油区极限位时的排量为 $16.32 \mathrm{~mL} / \mathrm{r}$, 计算得齿轮洜排量理论上可在 $88 \% \sim 100 \%$ 变化。 


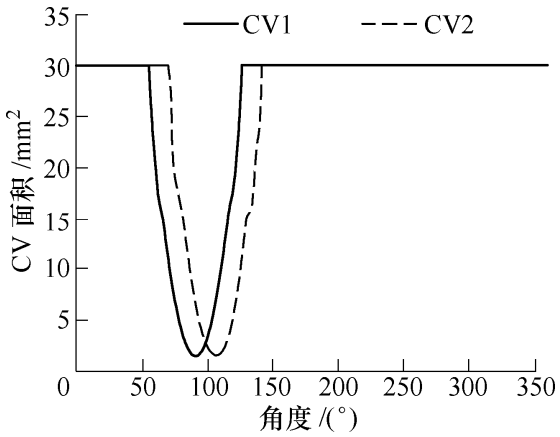

图 $6 \mathrm{CV} 1$ 与 $\mathrm{CV} 2$ 随角度的变化

\section{2 机液联合仿真分析}

根据前述齿轮葲参数及其三维模型, 在 SimualtionX 软件中搭建了如图 7 所示的联合仿真模 型。首先, 搭建单个齿轮腔模型, 然后依次搭建剩 余齿轮腔模型和变量模型, 构成变量齿轮洜液压系 统仿真模型; 并将测绘的齿轮百三维机械模型导入 软件中, 通过模块与液压系统构成变量齿轮泵机液 联合仿真模型。

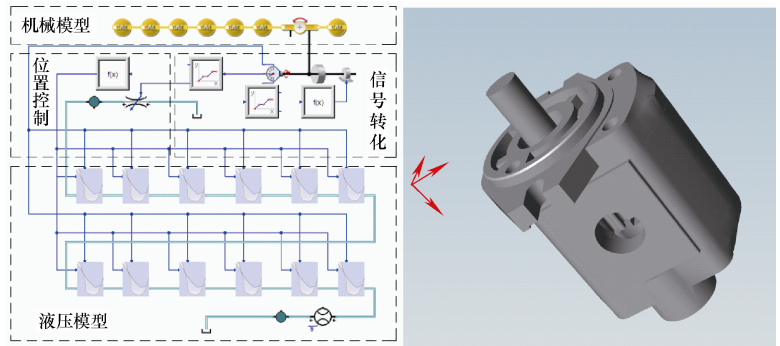

图 7 变量齿轮泵机液联合仿真模型

设定转速 $1000 \mathrm{r} / \mathrm{min}$, 负载压力 $5 \mathrm{MPa}$ 。为便 于理解, 提取了如图 8 所示的滑块位置不同时, 单 齿腔流量变化情况。由图 8 可知, 滑块位置由中位 向极限位移动时, 单齿腔流量会出现负值, 排出的 油液体积也会下降。而且随滑块位置偏移量增大, 流量负值增大，这是由于齿轮腔从高压区吸油体积 增大造成的，与理论分析结果一致。

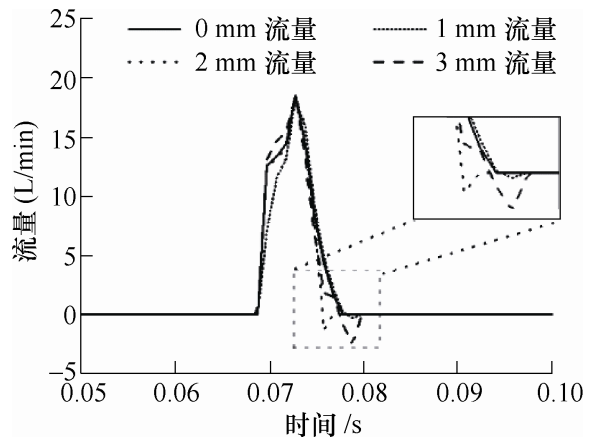

图 8 单齿腔流量仿真结果对比

图 9 所示为滑块在中位及极限位置时, 齿轮洜 的流量及排出油液体积曲线。通过计算, 齿轮泵在 中位时的平均流量约为 $17.56 \mathrm{~L} / \mathrm{min}$, 极限位时的平
均流量约为 $15.79 \mathrm{~L} / \mathrm{min}$, 仿真结果可知, 齿轮百排 量可在 $89 \% \sim 100 \%$ 变化。

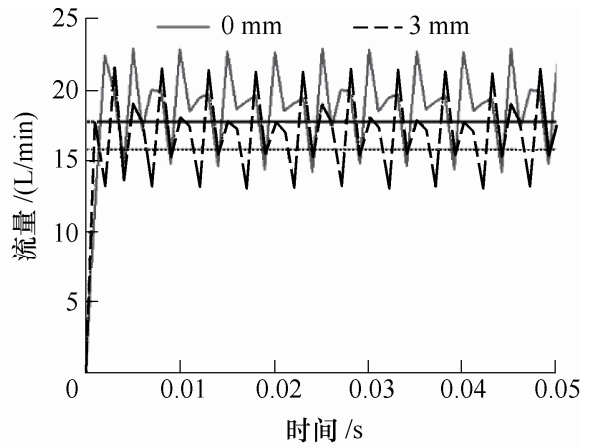

(a) 流量对比

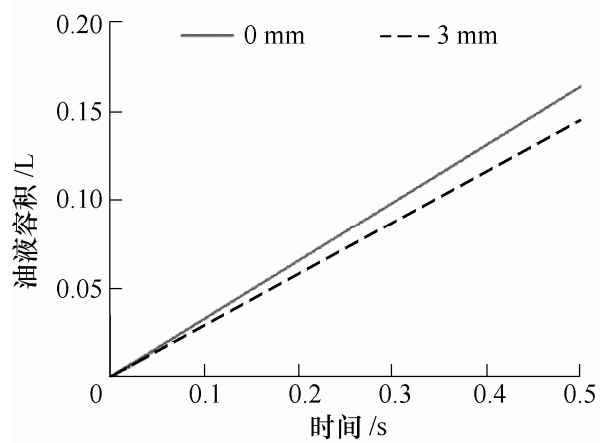

(b) 油液容积对比

图 9 整原输出流量仿真结果

仿真结果中流量脉动较大, 其原因是建模过程 中为计算便捷，将齿轮腔中将要排出齿腔的油液当 作已排出油液处理, 造成仿真结果流量脉动较大, 但不影响流量仿真结果均值, 如果在仿真模型中考 虑这部分因素，可减少流量脉动率。

\section{3 变量齿轮洜流场特性}

\section{1 数值模型}

在所建立的齿轮葲模型中全面考虑了齿轮洜中 的三种泄漏方式[齿顶径向间歇泄漏、齿面啮合处间 隙泄漏和齿轮端面间隙泄漏(由于齿轮端面与前后 盖之间间隙较大, 端面泄漏是齿轮泵中最主要的泄 漏方式，占总泄漏量的 $70 \% \sim 75 \%$ )] 和卸荷槽在内 的等等细节, 较为完整复原了整洜实际的流域体积。

通过比较仿真结果对网格的敏感性, 在设置网 格参数过程中, 确定了最大最小网格大小、边界处 的网格尺度以及特征角度，以限制所有单元格的最 小尺度，使网格模型在满足精度要求的同时减少计 算量, 加快仿真速度。针对不同体域的网格划分使 不同的, 进出口流体域为静流体域, 转子流体域是 动流体域, 动流域的网格是在仿真过程中不断变化 的，每一个时间步都将用插值方式形成新一步的网 
格模型以模拟真实齿轮啮合过程。并且不同流体域 之间需要设置相应匹配的数据传输边界(MGI), 使 得不同的流体域连接起来组成完整的整洜流域。在 保证计算精度的同时, 得到了在最优参数下的外啮 合齿轮㬌二叉树网格, 如图 10 所示。

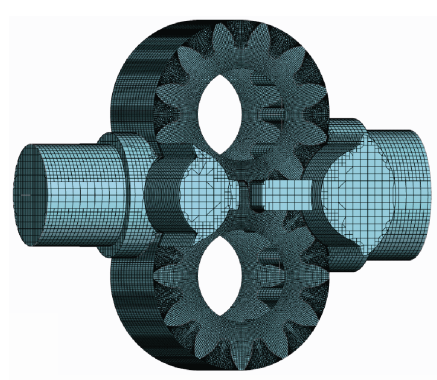

(a) 齿轮泵二叉树网络

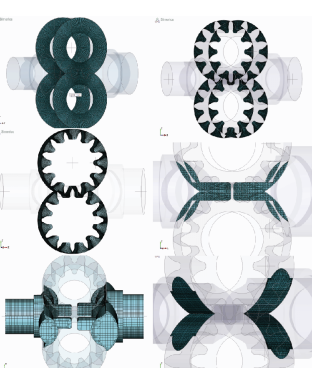

(b) 各流体域 (c) 不同体网格 网格示意间数据传输 交互面 (MGI)

图 10 外啮合齿轮泵二叉树网格

对齿轮百进行 CFD 数值模拟, 首先要建立一个 封闭的齿轮啮合流体域, 即主动齿轮和从动齿轮的 啮合流域呈现为一个完整的封闭连通体积, 使的两 齿轮间啮合线接触位置间隙足够小(不大于 0.04 $\mathrm{mm})$, 则认为此间没有流体流动, 如图 11 所示。

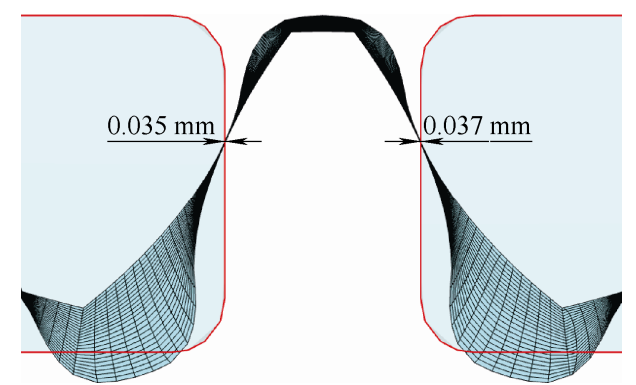

图 11 转子网格最小网格大小

齿轮百的变量方式主要是通过改变主动齿轮和 从动齿轮在啮合区困油面积大小来实现变量, 那么 对啮合极限点的定义变得尤为重要, 它将直接决定 滑块滑动的极限位置点。本研究设定在两极限位置 处, 两齿轮最小间隔大于 $0.05 \mathrm{~mm}$ 时, 视为两齿轮 分离。两极限点的选取如图 12 所示。

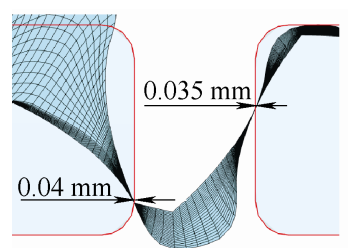

(a) 左极限位置网格大小

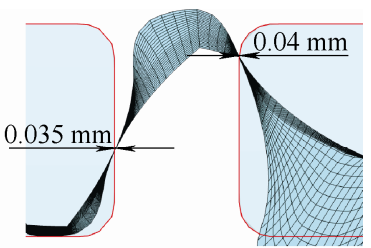

(b) 右极限位置网格大小
图 12 极限位置网格划分

\section{2 计算流体动力学控制方程}

计算流体流动现象模拟的本质是 Navier-Stokes (简称 N-S) 流动控制方程的求解, 式(10)、(11)为 N-S
的积分形式

$$
\begin{gathered}
\frac{\partial}{\partial t} \int_{\Omega(t)} \rho \mathrm{d} \Omega+\int_{\sigma} \rho\left(\boldsymbol{v}-\boldsymbol{v}_{\sigma}\right) \cdot \boldsymbol{n} \mathrm{d} \sigma=0 \\
\frac{\partial}{\partial t} \int_{\Omega(t)} \rho \boldsymbol{v} \mathrm{d} \Omega+\int_{\sigma} \rho\left[\left(\boldsymbol{v}-\boldsymbol{v}_{\sigma}\right) \cdot \boldsymbol{n}\right] \boldsymbol{v} \mathrm{d} \sigma= \\
\int_{\sigma} \tilde{\boldsymbol{\tau}} \cdot \boldsymbol{n} \mathrm{d} \sigma-\int_{\sigma} p \boldsymbol{n} \mathrm{d} \sigma+\int_{\Omega} \boldsymbol{f} \mathrm{d} \Omega
\end{gathered}
$$

式中, $\Omega$ 为计算域或控制体积, $\sigma$ 为 $\Omega$ 的表面, $n$ 为 $\sigma$ 的法线, $\rho$ 为流体密度, $p$ 表示压力, $f$ 为计算 域或控制体积所受的力, $v$ 为流体速度, $v_{\sigma}$ 为表面 运动速度。

本研究采用标准 $k-\varepsilon$ 湍流模型。在旋转类型的液 压泵中, 液体介质受粘性力和湍流的综合作用, 对 流体质点的速度和压力有强烈影响, 所以对这种在 高雷诺数下计算有效液体粘度的湍流模型是至关重 要的; 标准的 $k-\varepsilon$ 模型是一种高雷诺数的模型, 因其 适用范围广, 并有合理的计算精度, 所以在工业流 场和热交换模拟中有广泛应用。标准 $k-\varepsilon$ 模型的积分

$$
\begin{gathered}
\frac{\partial}{\partial t} \int_{\Omega(t)} \rho k \mathrm{~d} \Omega+\int_{\sigma} \rho\left[\left(\boldsymbol{v}-\boldsymbol{v}_{\sigma}\right) \cdot \boldsymbol{n}\right] \mathrm{kd} \sigma= \\
\int_{\sigma}\left(\mu+\frac{\mu_{t}}{\sigma_{k}}\right)(\nabla k \cdot \boldsymbol{n}) \mathrm{d} \sigma+\int_{\Omega}\left(G_{t}-\rho \varepsilon\right) \mathrm{d} \Omega \\
\frac{\partial}{\partial t} \int_{\Omega(t)} \rho \varepsilon \mathrm{d} \Omega+\int_{\sigma} \rho\left[\left(\boldsymbol{v}-\boldsymbol{v}_{\sigma}\right) \cdot \boldsymbol{n}\right] \varepsilon \mathrm{d} \sigma= \\
\int_{\sigma}\left(\mu+\frac{\mu_{t}}{\sigma_{\varepsilon}}\right)(\nabla \varepsilon \cdot \boldsymbol{n}) \mathrm{d} \sigma+\int_{\Omega}\left(c_{1} G_{t} \frac{\varepsilon}{k}-c_{2} \rho \frac{\varepsilon^{2}}{k}\right) \mathrm{d} \Omega
\end{gathered}
$$

式中, 经验常数为 $c_{1}=1.44, c_{2}=1.92, \sigma_{\mathrm{k}}=1, \sigma_{\varepsilon}=$ $1.3 ; k$ 为湍流动能; $\varepsilon$ 是湍流动能耗散率; $\mu$ 是湍流 黏度; $G_{\mathrm{t}}$ 湍流产生项。

计算机流体动力学仿真在划分网格和选择计算 模型后, 需要定义边界条件。转子转速为 $1000 \mathrm{r} / \mathrm{min}$ 。 模型设置转动 3 圈, 每转过一个齿模型计算 30 步, 每一步迭代步数为 500 步。其他边界如表 3 所示。

\section{表 3 模型边界条件}

\begin{tabular}{lc||lc}
\hline \multicolumn{1}{c|}{ 参数 } & 值 & \multicolumn{1}{c}{ 参数 } & 值 \\
\hline 油液密度 $/\left(\mathrm{kg} / \mathrm{m}^{3}\right)$ & 886 & 饱和压力 $/ \mathrm{MPa}$ & 0.004 \\
工作温度 $/ \mathrm{K}$ & 313 & 液体体积模量 $/ \mathrm{MPa}$ & 1500 \\
进口压力 $/ \mathrm{MPa}$ & 0.1 & 气体质量分数 & $9 \times 10^{-5}$ \\
出口压力 $/ \mathrm{MPa}$ & 5 & 气体相对分子质量 & 28.97 \\
动力黏度 $/(\mathrm{Pa} \cdot \mathrm{s})$ & $1.853 \times 10^{-5}$ & 蒸汽相对分子质量 & 300 \\
\hline
\end{tabular}

\section{3 结果与分析}

通过选择合理湍流和空化数值计算模型, 以及 验证二叉树网格敏感性, 选取最优网格对变量齿轮 泵进行了仿真分析，重点分析了滑块在不同位置时 原的输出流量变化, 与滑块在不同位置时负载压力 恒定情况下, 齿轮百主动齿轮齿腔和从动齿轮齿腔 
压力变化, 以及困油区流体速度变化。

\subsection{1 不同滑块位置输出流量对比}

图 13 所示为滑块在不同位置时的流量曲线。其 中, 中位代表困油区面积最小时的位置, 此时齿轮 泵单个齿腔可排出的有效体积最大; 左右位分别代 表滑块左右可移动的极限位置, 滑块左右极限位置 的齿腔可排出油液的有效体积要小于中位单个齿腔 可排出油液的有效体积, 所以左右极限位置的泵体 积流量为最小。仿真结果证实了不同滑块位置对应 不同的体积流量, 并且滑块从左到右移动过程, 泵 排量将由小变大, 在中位泵排量最大; 滑块从中位 继续向右移动时, 泵排量将由大变小。

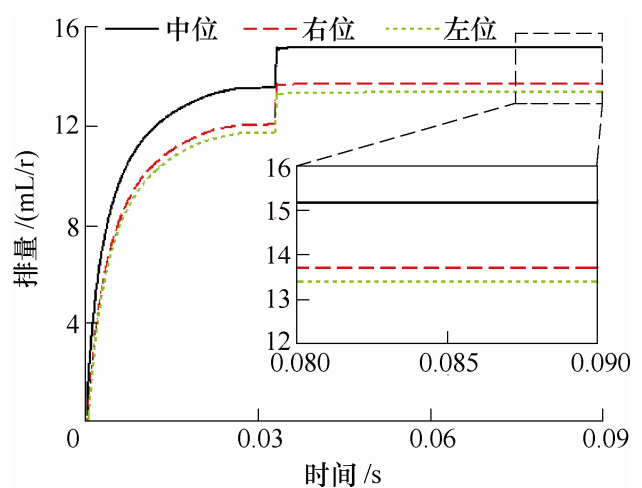

图 13 滑块不同位置时的流量对比

从图 13 可看出滑块在左右极限位置时泵体积 流量比较接近, 左极限位置情况下的排量略大于右 极限位置下的泵排量, 这是由于本研究采用的是负 变位齿轮, 左极限位置形成的困油区面积略大于右 极限位置所形成的困油区面积。

\subsection{2 齿腔压力分析}

从仿真结果看, 滑块从中间位置到左右两极限 位置都可以实现变量, 为了进一步确定齿轮洜选择 滑块移动方向, 在仿真模型的齿腔内设置了观测点, 通过对观测点设置用户自定义运动方程, 使观测点 与齿轮洜同步转动, 以测量齿轮百在转动过程中 主动齿轮和从动齿轮齿腔压力变化。观测点位置 如图 14a 所示, 滑块在三个不同位置的齿腔压力变 化分别如图 $14 b \sim 14 d$ 所示。

为使仿真结果收敛并趋于稳定预先仿真两圈, 取齿轮旋转第三圈时刻研究。从图 16 中可以看出, 滑块处于中位时, 观测点所在的主动齿轮腔在 $0.071 \mathrm{~s}$ 时刻与出口腔相连, 压力从进口压力迅速攀 升到负载压力; 在 0.0819 时, 由于齿轮啮合过程短 暂的困油, 致使齿腔压力出现峰值, 在下一时刻, 困油区消失, 油液倒泄到吸油腔后压力骤减。滑块 处于左右极限位置时, 压力变化趋势相同, 但在困 油区的压力峰值明显不同, 滑块在右极限位置时困
油压力略低于中位困油压力, 而滑块处于左极限位 置时困油压力激增到 $9.27 \mathrm{MPa}$ 。较高的困油压力会 导致径向不平衡力和噪声的增加。这是在设计齿轮 过程中严格避免的情况，则应选择滑块右移来实现 齿轮百变量。

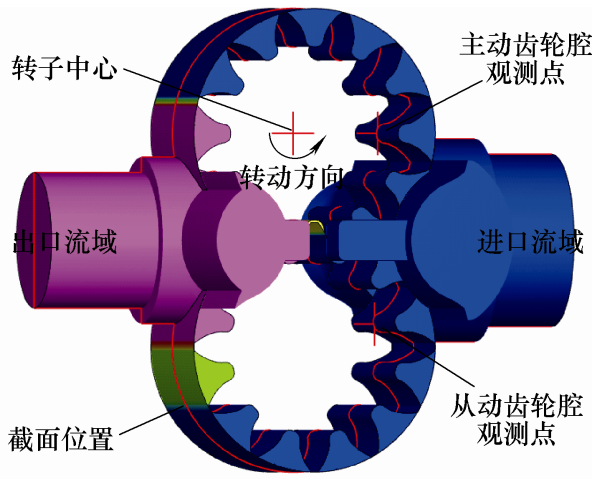

(a) 观测点和截面位置定义

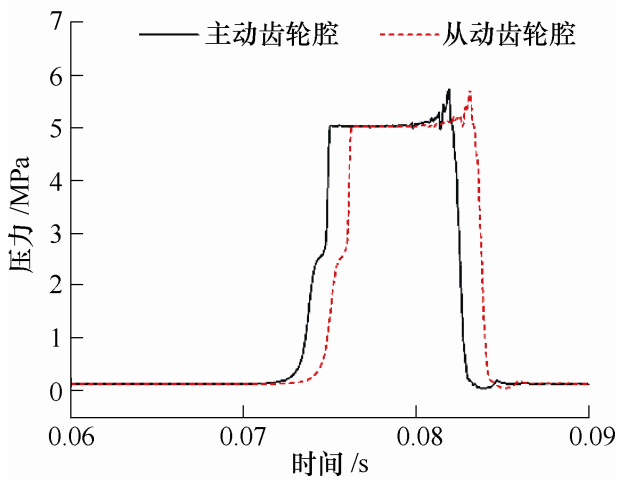

(b) 中间位置齿腔压力变化

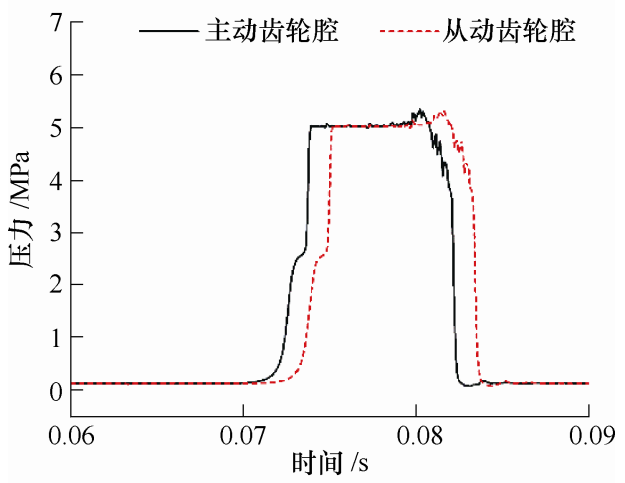

(c) 右极限位置齿腔压力变化

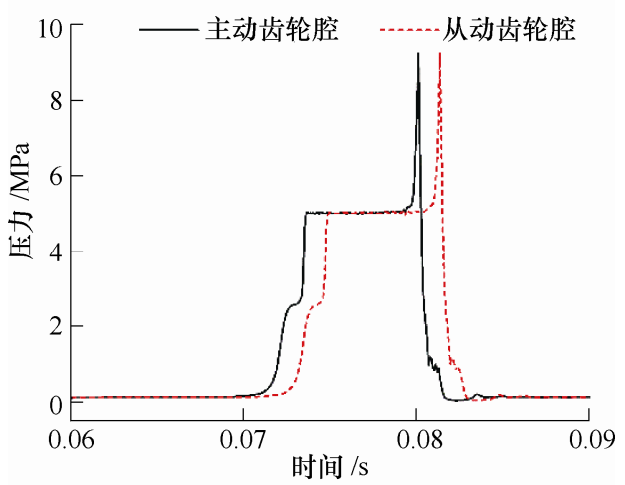

(d) 左极限位置齿腔压力变化

图 14 滑块处在不同位置时, 齿轮泵旋转一周齿腔压力变化 


\subsection{3 困油区压力与速度分析}

以主动齿轮观测点所在的齿轮腔为研究对象, 研究观测点所在齿腔从进入啮合到啮合结束过程中 的齿腔压力变化和油液流速变化。下图中旋转角 $\theta$ 是观测点从初始位置开始随齿腔旋转过的角度。

滑块处于中间位置时困油区压力云图和流体速 度矢量图如图 15 所示。主动齿轮观测点所在齿腔从 $\theta=256^{\circ}$ 开始进入啮合, 在 $\theta=285^{\circ}$ 时啮合结束。在刚 进入啮合时, 此齿腔与出油口卸荷槽相连, 齿腔压 力近似等于负载压力; 当 $\theta=263^{\circ}$ 时, 此齿腔逐渐减 小并脱离卸荷槽, 此时齿腔压力值如图 $16 \mathrm{~b}$ 所示达 到峰值, 由于齿间间隙的存在, 油液从右侧齿间隙 射流到了低压区, 从图 15 中可知射流速度要远高于 齿轮转速; 随着齿腔压力逐渐减小, 直到在 $\theta=263^{\circ}$ 时, 齿腔脱离困油区而与低压卸荷槽相连, 齿腔压 力降低到最小值。

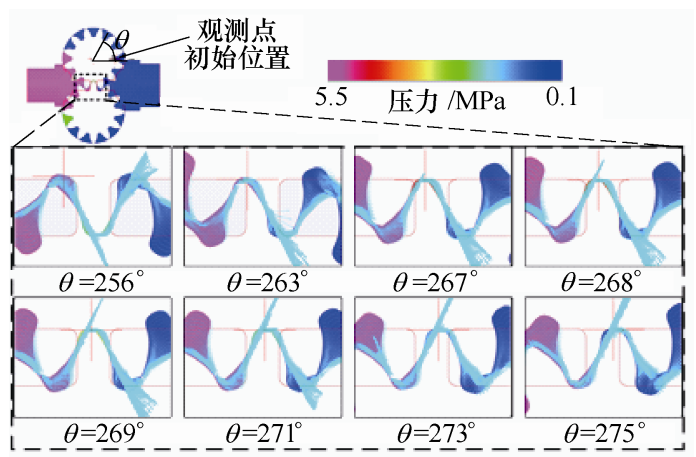

图 15 滑块处于中间位置时, 截面处压力 云图和流体速度矢量图

滑块处于右极限位置时困油区压力云图和流体 速度矢量图如 16 所示。主动齿轮观测点所在齿轮腔 从 $\theta=243^{\circ}$ 开始进入啮合, 在 $\theta=271^{\circ}$ 时啮合结束。齿 轮旋转角 $\theta$ 从 $243^{\circ}$ 到 $257^{\circ}$ 过程中, 观测点所在的齿 腔齿腔面积不断减小, 但由于整个过程中齿腔与出 油口卸荷槽相连, 并在右侧齿间间隙伴有间隙泄漏, 所以齿腔压力基本保持平稳; 随着齿轮旋转, 齿腔 与出油口卸荷槽接触面积逐渐减小, 而齿腔面积逐 渐增大, 所以在困油区不会造成齿腔压力增加。因 此齿腔可以平稳从困油区过渡到低压区, 减少对进 油口的压力冲击。

滑块处于左极限位置时困油区压力云图和流体 速度矢量图如 17 所示。主动齿轮观测点所在齿轮腔 从 $\theta=239^{\circ}$ 开始进入啮合, 在 $\theta=268^{\circ}$ 时啮合结束。从 图中可以看出, 观测点所在齿腔在经过困油区的整 个过程中, 齿腔面积都在逐渐减小; 在 $\theta=241^{\circ}$ 时, 由主动齿轮、从动齿轮以及滑块形成困油死区, 齿 腔油液受到挤压而压力达到峰值, 并且此时油液射 速速度相比中位和右极限位的油液射流速度更大,
齿腔将更容易发生空化现象，齿轮壁面将更容易受 到冲蚀。这也进一步印证了应该选用 “右移” 为滑 块变量机构的变量方式。

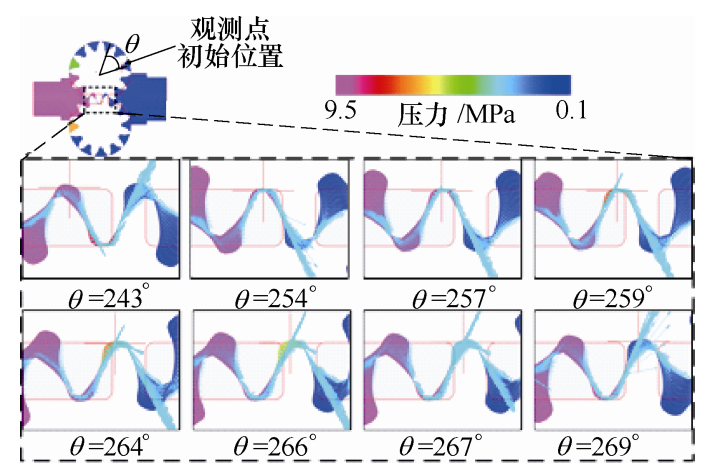

图 16 滑块处于右极限位置时, 截面处压力 云图和流体速度矢量图

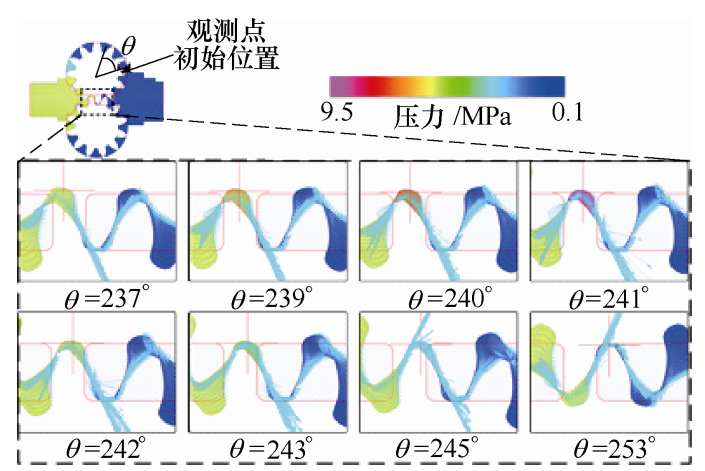

图 17 滑块处于左极限位置时, 截面处压力 云图和流体速度矢量图

\section{4 试验结果分析}

为验证理论和仿真分析结果, 搭建了齿轮洜试 验台, 图 18 所示为试验原理, 采用压力传感器检测 齿轮葲出口压力, 流量传感器检测齿轮洜出口流量, 溢流阀用于加载。

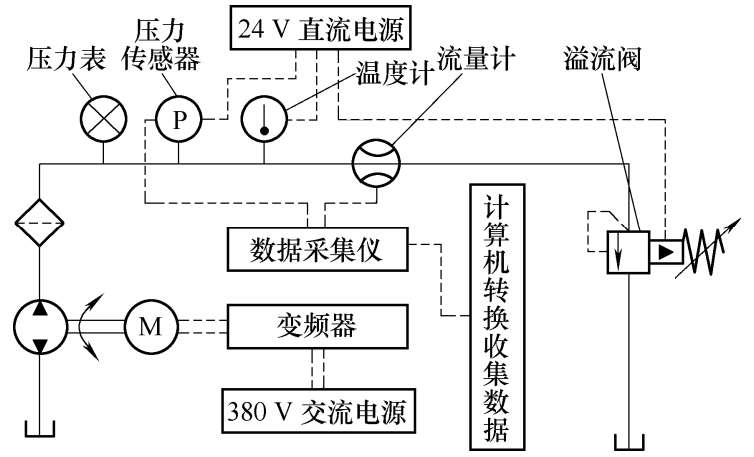

图 18 试验系统搭建

为对齿轮百变量原理进行验证, 制造了模拟滑 块处于中位和极限位置的轴套, 图 19 所示为试验照 片及制造的轴套照片。选用 ISO VG46 液压油(油液密 度 $846.9 \mathrm{~kg} / \mathrm{m}^{3}$ 进行试验。调定电机转速为 $1000 \mathrm{r} / \mathrm{min}$, 
外负载压力 $5 \mathrm{MPa}$ 。

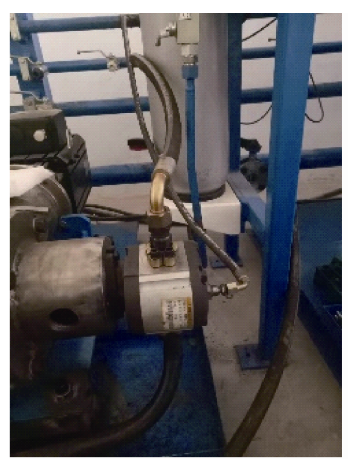

(a) 试验用齿轮百

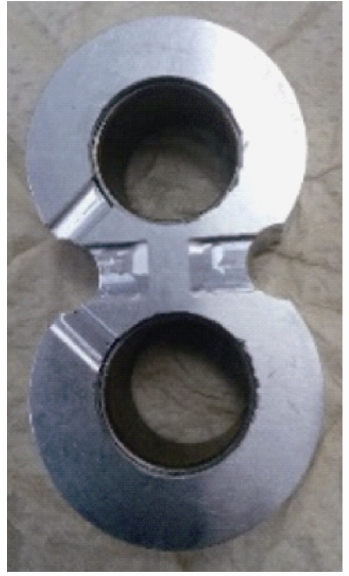

(c) 中位轴套

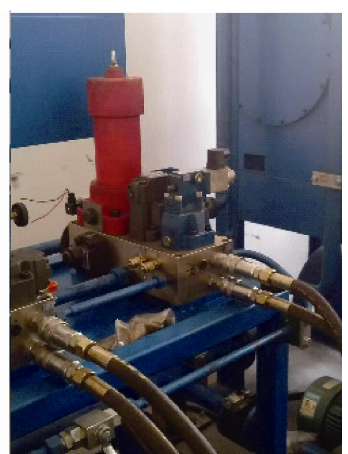

(b) 加载用溢流阀

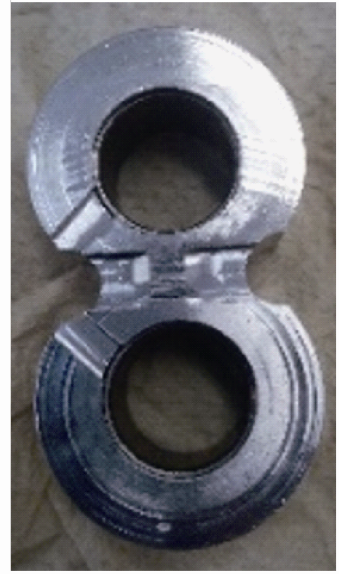

(d) 极限位轴套
图 19 试验照片

图 20 所示为滑块不同位置时, 齿轮泵的流量压 力试验测试曲线。通过测试发现, 该齿轮洜在其他 测试条件不变的情况下, 通过改变滑块位置, 便可

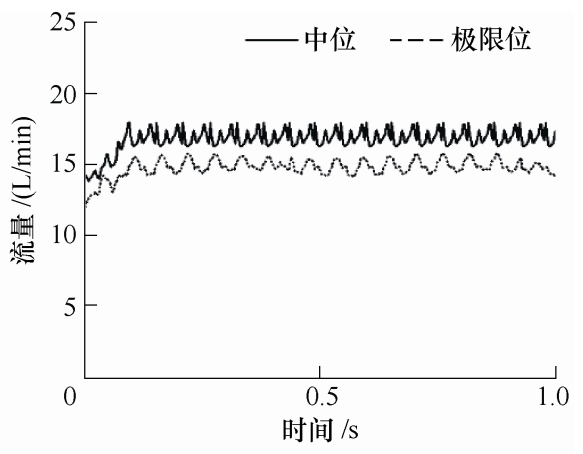

(a) 流量对比

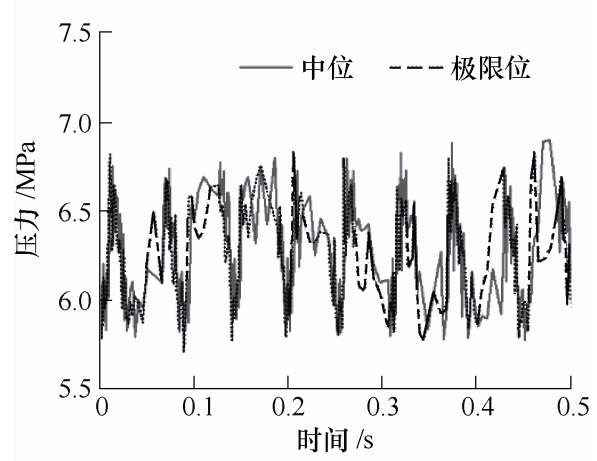

(b) 压力对比

图 20 中位与极限位试验结果对比
实现齿轮百的变量。经测试, 滑块处于中位时, 平 均流量约为 $16.92 \mathrm{~L} / \mathrm{min}$; 处于极限位时, 平均流量 约为 $14.54 \mathrm{~L} / \mathrm{min}$, 齿轮洜变量范围是 $86 \% \sim 100 \%$ 同时，两位置时齿轮洜出口处压力曲线相似，证实 该齿轮泵实际使用过程的安全性, 为将来变量齿轮 的实际使用奠定基础。

变量齿轮百的变量效果与极限位时困油面积大 小有关, 通过改变齿轮洜齿形结构(在原有齿轮百齿 轮基础上, 将齿轮洜齿轮加工为非对称轮齿等方 式), 调整极限位困油面积可以提高变量效果。

\section{5 结论}

过理论分析、仿真和试验, 对变量齿轮洜特性 进行了深入研究, 得到以下结论。

(1) 提出机液联合仿真宏观特性和流场可视化 仿真微观特性相结合的方法, 为齿轮泵的仿真研究 提供了新途径。

(2) 提出一种变滑块位置一困油区域的齿轮百 变量方式, 实现齿轮泵排量小范围调节。结果表明, 所提原理可实现齿轮洜排量在 $88 \% \sim 100 \%$ 调节。该 变量齿轮洜实现了齿轮百排量小范围变化, 可用于 解决洜控单出杆缸系统有杆腔与无杆腔流量不匹配 问题。

在未来的工作中，将对变量机构进行设计和优 化, 实现齿轮洜排量的自动调节; 进一步分析齿轮 齿形等参数对齿轮百变量范围的影响, 优化设计齿 轮齿形，实现更大排量的调节。

\section{参 考 文 献}

[1] MOČILAN M, HUSÁR Š, LABAJ J, et al. Non-stationary CFD simulation of a gear pump $[\mathrm{J}]$. Procedia Engineering, 2017, 177: 532-539.

[2] 李玛璠, 赵斌, 张晓刚, 等. 基于 SimulationX 的外齿 轮洜输出特性的研究 [J]. 液压与气动, 2018(11): 13-17. LI Yufan, ZHAO Bin, ZHANG Xiaogang, et al. Research on output characteristics of external gear pump with simulationX[J]. Chinese Hydraulics \& Pneumatics, 2018 (11): 13-17.

[3] FROSINA E, SENATORE A, RIGOSI M. Study of a high-pressure external gear pump with a computational fluid dynamic modeling approach[J]. Energies, 2017, 10(8): 1113.

[4] DEL CAMPO D, CASTILlA R, RAUSH G A, et al. Pressure effects on the performance of external gear pumps under cavitation[J]. Proceedings of the Institution of Mechanical Engineers, Part C: Journal of Mechanical 
Engineering Science, 2014, 228(16): 2925-2937.

[5] RITURAJ F, VACCA A. External gear pumps operating with non-Newtonian fluids: Modelling and experimental validation[J]. Mechanical Systems and Signal Processing, 2018, 106: 284-302.

[6] VACCA A, FRANZONI G, CASOLI P. On the analysis of experimental data for external gear machines and their comparison with simulation results[C]//ASME 2007 International Mechanical Engineering Congress and Exposition. American Society of Mechanical Engineers, 2007: 45-53.

[7] CASOLI P, VACCA A, FRANZONI G. A numerical model for the simulation of external gear pumps $[\mathrm{C}] / /$ Proceedings of the JFPS International Symposium on Fluid Power. The Japan Fluid Power System Society, 2005, 2005(6): 705-710.

[8] YOON Y, PARK B H, SHIM J, et al. Numerical simulation of three-dimensional external gear pump using immersed solid method[J]. Applied Thermal Engineering, 2017, 118: 539-550.

[9] MUCCHI E, DALPIAZ G, RIVOLA A. Elastodynamic analysis of a gear pump. Part II: Meshing phenomena and simulation results[J]. Mechanical Systems and Signal Processing, 2010, 24(7): 2180-2197.

[10] MUCCHI E, RIVOLA A, DALPIAZ G. Modelling dynamic behaviour and noise generation in gear pumps: Procedure and validation[J]. Applied Acoustics, 2014, 77 : 99-111.

[11] 阮学云, 刑佑亭, 钮炳瑜. 基于频谱分析的 CBK 型齿 轮泵噪声诊断及控制 [J]. 机床与液压，2012，40(9): 147-150.

RUAN Xueyun, XING Youting, NIU Bingyu. Noise diagnosis and control for CBK gear pump based on spectrum analysis[J]. Machine Tool \& Hydraulics, 2012, 40(9): 147-150.

[12] 杜沙沙. 外啮合齿轮洜模型建立以及振动和噪声研究[D]. 葫芦岛：辽宁工程技术大学, 2012 .

DU Shasha. External gear pump model establishment and the research of vibration and noise[D]. Huludao: Liaoning Technical University, 2012.

[13] 高彦军, 谷立臣, 焦龙飞. 基于油液特征模型的外啮 合齿轮洜数值仿真分析 $[J]$. 机械设计与制造, 2017(8): 209-212.

GAO Yanjun, GU Lichen, JIAO Longfei. Numerical simulation analysis of external gear pump based on oil characteristic model[J]. Machinery Design \& Manufacture, 2017(8): 209-212.

[14] 李明学. 渐开线外啮合直齿轮泵困油现象分析与研究[D]. 兰州理工大学, 2016 .
LI Mingxue. Analysis and research on oil-trapping phenomenon of involute external meshing spur gear pump[D]. Lanzhou: Lanzhou University of Technology, 2016.

[15] 李玉龙. 基于低速困油模型的外啮合齿轮泵高速困油 特性分析[J]. 农业工程学报，2012，28(9)：35-39.

LI Yulong. Characteristics analysis of high-speed trapped-oil in external gear pump based on low-speed trapped-oil model[J]. Transactions of the Chinese Society of Agricultural Engineering， 2012， 28(9): 35-39.

[16] 李玉龙, 刘焜. 外啮合齿轮泉困油面积和卸荷面积计算 式的建立[J]. 农业机械学报，2009，40(6)：203-207.

LI Yulong, LIU Kun. Established formulas for trapped-oil area and relief-load area of external spur-gear pump[J]. Transactions of the Chinese Society for Agricultural Machinery, 2009, 40(6): 203-207.

[17] 吴小锋, 刘春节, 干为民, 等. 面向外啮合齿轮葲困 油问题的健壮性设计 [J]. 航空动力学报, 2015, 30(11): 2721-2729.

WU Xiaofeng, LIU Chunjie, GAN Weimin, et al. Robust design of external gear pump with trapped oil problem[J]. Journal of Aerospace Power, 2015，30(11): 2721-2729.

[18] 藏克江, 周欣, 顾立志, 等. 降低齿轮原困油压力新 方法的研究 [J]. 中国机械工程, 2004, 15(7): 578-581. ZANG Kejiang, ZHOU Xin, GU Lizhi, et al. Study on new method of reducing the trap pressure of gear pump[J]. China Mechanical Engineering, 2004, 15(7): 578-581.

[19] 杜睿龙, 谢安桓, 周华, 等. 基于集中参数法的内啮合齿 轮洜仿真[J]. 华中科技大学学报, 2018, 46(9): 101-106. DU Ruilong, XIE Anhuan, ZHOU Hua, et al. Simulation on internal gear pumps utilizing lumped parameter approach[J]. Journal of Huazhong University of Science and Technology, 2018, 46(9): 101-106.

[20] 吴晓明, 王小鹏, 马立瑞. 基于集中参数法内啮合齿轮 原 AMESim 模型的建模方法与仿真研究 [J]. 液压与气 动, 2017 (7): 103-109.

WU Xiaoming, WANG Xiaopeng, MA Lirui. Modeling and simulation based on lumped parameter method by AMESim for internal gear pump[J]. Chinese Hydraulics \& Pneumatics, 2017(7): 103-109.

[21] CASTILlA R, GAMEZ-MONTERO P J, DEL CAMPO $D$, et al. Three-dimensional numerical simulation of an external gear pump with decompression slot and meshing contact point [J]. Journal of Fluids Engineering, 2015, 137(4): 041105 .

[22] 李阁强, 张龙飞, 韩伟锋, 等. 双圆弧斜齿齿轮泉脉动 特性分析及齿形设计 $[\mathrm{J}]$. 中国机械工程，2018，29(2): 186-192.

(下转第 252 页) 
Information Entropy[J]. Journal of Computer Research and Development, 2016, 53(5): 1019-1027.

[20] ALAYKYRAN K, ENGIN O, DOYEN A. Using ant colony optimization to solve hybrid flow shop scheduling problems[J]. The International Journal of Advanced Manufacturing Technology, 2007, 35(5-6): 541-550.

[21] NERSON E, BAPTISTE P, GUPTA J N D. Solving hybrid flow shop problem using energetic reasoning and global operations[J]. Omega, 2001, 29(6): 501-511.

[22] SANTOS D L, HUNSUCKER J L, DEAL D E. Global lower bounds for flow shop with multiple processors[J]. European Journal of Operational Research，1995，80(1): 112-120.

[23] 徐华, 张庭. 混合离散蝙蝠算法求解多目标柔性作业车
间调度[J]. 机械工程学报，2016，52(18): 201-211.

XU Hua, ZHANG Ting. Hybrid discrete bat algorithm for solving the multi-objective flexible job shop scheduling problem[J]. Journal of Mechanical Engineering, 2016, 52(18): 201-211.

作者简介: 韩忠华, 男, 1977 年出生, 博士, 硕士研究生导师。主要研 究方向为生产与运作管理、企业自动化系统集成技术、车间排产与生产 调度算法的工程应用。

E-mail: hanzhonghua@sia.cn

张权(通信作者), 男, 1994 年出生, 硕士。主要研究方向为车间排产与 生产调度算法的工程应用。

E-mail: zhangq0716@163.com

史海波, 男, 1966 年出生, 博士, 研究员, 博士研究生导师。主要研究 方向为生产与运作管理、企业自动化系统集成技术、车间排产与生产调 度算法的工程应用。

E-mail: hbshi@sia.cn

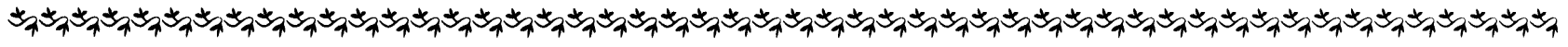

\section{(上接第 235 页)}

LI Geqiang, ZHANG Longfei, HAN Weifeng, et al. Pulsation characteristic analysis and tooth profile design of double-circular-arc helical gear pumps[J]. China Mechanical Engineering, 2018，29(2): 186-192.

[23] 孙浩林. 基于圆弧齿形的低脉动齿轮泉特性研究[D]. 哈 尔滨：哈尔滨工业大学, 2018.

SUN Haolin. Research on low-pulse external gear pump based on circular-arc gear profile[D]. Harbin: Harbin Institute of Technology, 2018.

[24] DEVENDRAN R S, VACCA A. A novel design concept for variable delivery flow external gear pumps and motors[J]. International Journal of Fluid Power, 2014, 15(3): 121-137.

[25] VACCA A, DEVENDRAN R S. A flow control system for a novel concept of variable delivery external gear pump $[\mathrm{C}] / 10$ th International Fluid Power Conference-Dresden. 2016: 263-274.

[26] 杨德贵, 钟定莲. 径向移动变量齿轮不 (马达): 中国, 85109203[P]. 1987-06-03.
YANG Degui, ZHONG Dinglian. Radial-movable variable displacement gear pump (Motor): China, 85109203[P]. 1987-06-03.

[27] WINMILL L. Adjustable-displacement gear pump: U.S., 09/734, 326[P]. 2001-09-27.

[28] 闻德生, 商旭东, 潘为圆, 等.齿轮型多泵多马达传动 系统设计与试验 $[\mathrm{J}]$. 农业机械学报，2017，48(6): 399-406.

WEN Desheng, SHANG Xudong, PAN Weiyuan, et al. Design and experiment on gear multi-pump and multi-motor driving system[J]. Transactions of the Chinese Society for Agricultural Machinery, 2017, 48(6): 399-406.

作者简介: 赵斌, 男, 1981 年出生, 博士, 副教授, 硕士研究生导师。 主要从事机电装备建模理论及智能控制、电液节能系统、液压元件等方 面的研究。

E-mail: zbtyut@126.com

权龙(通信作者), 男, 1959 年出生, 博士，教授，博士研究生导师。主 要研究方向为电液伺服及比例控制技术, 工程机械节能。

E-mail: quanlong@tyut.edu.cn 